\title{
EchoGéo
}

$47 \mid 2019$

Nouvelles géographies de la collecte

\section{« Faire la ferraille » en banlieue parisienne : glaner, bricoler et transgresser}

\section{Bénédicte Florin et Pascal Garret}

\section{(2) OpenEdition}

1 Journals

Édition électronique

URL : https://journals.openedition.org/echogeo/16942

DOI : $10.4000 /$ echogeo. 16942

ISSN : 1963-1197

Éditeur

Pôle de recherche pour l'organisation et la diffusion de l'information géographique (CNRS UMR 8586)

Référence électronique

Bénédicte Florin et Pascal Garret, « « Faire la ferraille » en banlieue parisienne : glaner, bricoler et transgresser », EchoGéo [En ligne], 47 | 2019, mis en ligne le 21 avril 2019, consulté le 10 août 2021.

URL : http://journals.openedition.org/echogeo/16942 ; DOI : https://doi.org/10.4000/echogeo.16942

Ce document a été généré automatiquement le 10 août 2021.

EchoGéo est mis à disposition selon les termes de la licence Creative Commons Attribution - Pas d'Utilisation Commerciale - Pas de Modification 4.0 International (CC BY-NC-ND) 


\title{
«Faire la ferraille » en banlieue parisienne : glaner, bricoler et transgresser
}

\author{
Bénédicte Florin et Pascal Garret
}

\author{
V'la le drillou, cherchez partout, \\ Inspectez, puis vendez surtout : \\ Vieux bouquins, bassines, étrilles, \\ Vieilles platines, vieux étain ; \\ J'achète les vieux ustensiles, \\ Rosette, cuivre, plomb, airain, \\ Puis couteaux, cuisinières, coquilles, \\ Tourne-broche, pochins, grille-pain \\ J'achète râpes, réchauds grilles, \\ Les fanfreluches inutiles \\ Et les paltoquets de vilains; \\ J'achète habits vieux et drilles, \\ Casquettes, sans-souci, mantilles, \\ Livres classiques et calpins. \\ Puis enfin les peaux de lapins. \\ Pierre Brivot, 1874, [extrait].
}

\section{Introduction : Du ferraillou au ferrailleur}

1 Le "ferraillou ", terme argotique un peu moqueur, parfois vaguement méprisant, désigne le récupérateur de ferraille qui parcourt les rues des grandes villes et des banlieues à la recherche de métaux ferreux ou non ferreux. En 1874, Pierre Brivot, chiffonnier à Mâcon, rédigea un poétique opuscule, Le chant patriotique du drillou, du ferraillou et du chiffonnier, où un long inventaire à la Prévert énumérait tout ce qui était récupérable: des vieux papiers aux peaux de lapins ${ }^{1}$ en passant par des matériaux nobles tels que le cuivre, l'étain, l'airain, le bronze et le platine. Les ferraillous se 
distinguent des "ferrailleurs ", grossistes alimentant en matières premières secondaires les aciéries et fonderies. Quant à l'expression "faire la ferraille ", elle renvoie à l'activité de récupération des matériaux dans l'espace public, même si certains patrons l'emploient pour qualifier leur propre activité. D'ailleurs, les ferrailleurs ont souvent commencé leur activité comme ferraillous: l'Italien Luigi Costella, migrant à Paris en 1915, a débuté comme chiffonnier dans la "Zone » avant de développer l'entreprise de ferraille, aujourd'hui dirigée par son arrière-petit-fils ${ }^{2}$; la société Serres \& Andrieu a été fondée par le grand-père du directeur actuel qui avait commencé à Sarcelles, dans les années 1920, par l'achat de peaux de lapin dans des fermes, avant de se lancer dans la ferraille (entretien BF \& PG, Genneviliers, 06/06/20183). Et l'on peut encore citer ici Guy Derichebourg qui, investissant en 1958 dans une Estafette Renault, collectait la ferraille au porte-à-porte avant d'élargir ses activités et de devenir la prospère entreprise éponyme ${ }^{4}$.

2 Traditionnellement considéré comme inscrit dans une économie de la pauvreté, le ferraillou exerce un «job de la débrouille $»^{5}$. Pourtant, il mobilise des outils, des moyens de transport, un investissement financier et un capital relationnel importants. Faire la ferraille se distingue ainsi de la collecte dans les poubelles (Corteel et Le Lay, 2011 ; Cirelli et Florin, 2015) et de la biffe (Milliot, 2010 et 2011 ; Balan, 2014) et ceux qui la pratiquent ne sont pas toujours les plus démunis. Même si elle imbrique au secteur formel des pratiques informelles, cette activité n'en alimente pas moins le marché mondial des métaux et la valeur de la ferraille est alignée sur les cours de ce marché spéculatif et lucratif. Les grossistes ferrailleurs réalisent jusqu'à $50 \%$ de leur chiffre d'affaire grâce aux apports des ferraillous qui ne représentent pourtant que $30 \%$ de leur approvisionnement: c'est la valeur des métaux et le travail réalisé sur les matériaux qui explique ce différentiel. L'économie de la ferraille, reposant en partie sur l'activité des ferraillous, est donc fortement rémunératrice pour les intermédiaires que sont les ferrailleurs. Sans être pleinement reconnue, elle s'inscrit dans l'économie circulaire, tant prônée par ailleurs, ainsi que dans des filières mondialisées du recyclage.

\section{Dans les marges, des cueilleurs marginaux?}

3 Ces récupérateurs de ferraille dans les rues, les chantiers de démolition ou les bâtiments abandonnés peuvent être considérés comme des «cueilleurs contemporains ». Du fait de leur connaissance de la ville et de ses modes de fonctionnement, leur cueillette est loin d'être hasardeuse et la ville, si elle leur oppose des obstacles, leur offre aussi quantités de ressources. Faire la ferraille se décline dans des lieux particuliers où le récupérateur peut trier, découper, classer ses trouvailles avant de les revendre: des garages, des bidonvilles, des friches (péri)urbaines, des dents creuses ou même des sites de relogement. Autant d'interstices discrets et de marges urbaines où se déploie cette activité difficilement contrôlable. Or, ces « espaces de la ferraille ", opportunités spatiales indispensables à la poursuite de l'activité, sont de plus en plus menacés par la rénovation urbaine, déjà en cours sur la Plaine SaintDenis ou en projet pour les Jeux Olympiques de 2024.

4 À la suite de nos entretiens et observations menés dans les banlieues nord, est et sud de Paris $^{6}$, nous interrogeons ici la "marginalité » de cette cueillette, des lieux qu'elle occupe, mais également des hommes et des femmes qui la pratiquent, souvent 
considérés comme en périphérie de la société, en particulier lorsqu'il s'agit de populations considérées comme marginales : le « Rom " ${ }^{7}$, l'exilé clandestin, la personne en situation de précarité économique. Par leurs pratiques et savoir-faire, les récupérateurs de ferraille utilisent les marges comme des espaces ressources, mais tentent aussi de s'affranchir de leur mise à la marge sociale et économique.

\section{Entretiens et portraits : culture du silence versus demande de reconnaissance}

5 En 2016, nous avons initié plusieurs campagnes d'entretiens sur la question du travail avec des Roms roumains installés dans des sites d'hébergement à Ivry-sur-Seine et Saint-Denis où les municipalités ont relogé des familles venues des bidonvilles (Cousin et Legros, 2014) $)^{8}$. À partir de 2017, la recherche s'est enrichie d'une dizaine d'entretiens réalisés avec des patrons-ferrailleurs à Aubervilliers, La Courneuve, Saint-Ouen, Bonneuil-sur-Marne et Gennevilliers. La culture de la discrétion, sinon du secret, reste cependant très prégnante chez ces derniers et nous avons parfois été rudement congédiés, mais ceux qui ont finalement accepté de témoigner, l'ont fait longuement. Ce que nous transcrivons ici renvoie surtout aux discours et représentations que ces ferrailleurs portent sur les ferraillous. Cette nouvelle investigation nous a aussi permis de discuter avec des particuliers qui attendent leur tour avant de vendre leur collecte dans les cours de ces ferrailleurs. Ces entretiens, parfois limités par l'activité intense qui prévaut, ont été menés avec des personnes en situation de précarité sociale, économique et, souvent, administrative : des Roms de Roumanie, Hongrie et Bulgarie, un Malien sans-papiers, rencontré à plusieurs reprises, et d'autres récupérateurs de ferraille. Une vingtaine d'entretiens ont été conduits afin de reconstituer les parcours des ferraillous, leurs pratiques et circulations ainsi que leurs relations avec les ferrailleurs. ${ }^{9}$

6 La première partie de cet article s'attache aux hommes et femmes qui font la ferraille afin de saisir les modalités de leur apprentissage et leurs expériences. Les itinéraires des trois figures choisies ici, celle de l'exilé sans-papiers, du Rom et du Français, témoignent qu'il s'agit bien d'une économie de la pauvreté, même si les positions sociales différentes des uns et des autres influent sur les revenus qu'ils en tirent.

7 La seconde partie s'intéresse aux pratiques professionnelles des ferraillous qui savent où collecter la ferraille et les encombrants, qui bricolent d'originaux attelages lorsqu'ils n'ont pas de camionnette et qui travaillent sur les matériaux récupérés afin d'en augmenter la valeur. Si ramasser un objet déposé sur la voie publique n'est pas interdit en France, les récupérateurs rencontrent toutefois des obstacles qu'ils contournent en adoptant des tactiques spécifiques que l'on peut lire comme autant de discrètes transgressions. Enfin, si faire la ferraille leur permet, dans une certaine mesure, d'échapper à la précarité et de réduire leur marginalisation, il n'en reste pas moins qu'ils demeurent aux lisières du marché du travail formel. Pour ce qui concerne les Roms - ou les personnes assimilées dans les représentations communes à des Roms -, la construction sociale du regard porté sur eux les cantonne dans une catégorie à part, d'autant plus lorsqu'ils sont vus en train de faire la ferraille, pratique jugée déviante. 


\section{Faire la ferraille : « un job de la débrouille»}

\section{L'apprentissage ou la circulation de l'expérience}

8 N'importe qui, à condition d'être en bonne forme physique, peut arpenter les rues des villes, ou plus souvent de leurs banlieues, pour y récupérer des matériaux revendables : il n'existe pas de formation spécifique pour cette activité que l'on peut apprendre «sur le tas », en observant et en discutant avec ceux qui la pratiquent. Parti de son village du Mali « où il n'y avait plus rien à manger ", Sidiki a traversé la Libye, la Méditerranée, puis l'Italie, pour arriver en 2013 en banlieue nord, hébergé par une connaissance. Cherchant à gagner sa vie, il remarque les va-et-vient des récupérateurs devant un ferrailleur et, demandant conseil à certains, il comprend comment fonctionne le travail et, surtout, qu'il peut l'exercer sans avoir à demander d'autorisation à quiconque (PG, 28/06/2016, 01/12/2016 et 05/06/2018).

Illustration 1 - Sidiki arrivant chez le ferrailleur à la fin d'une tournée de collecte

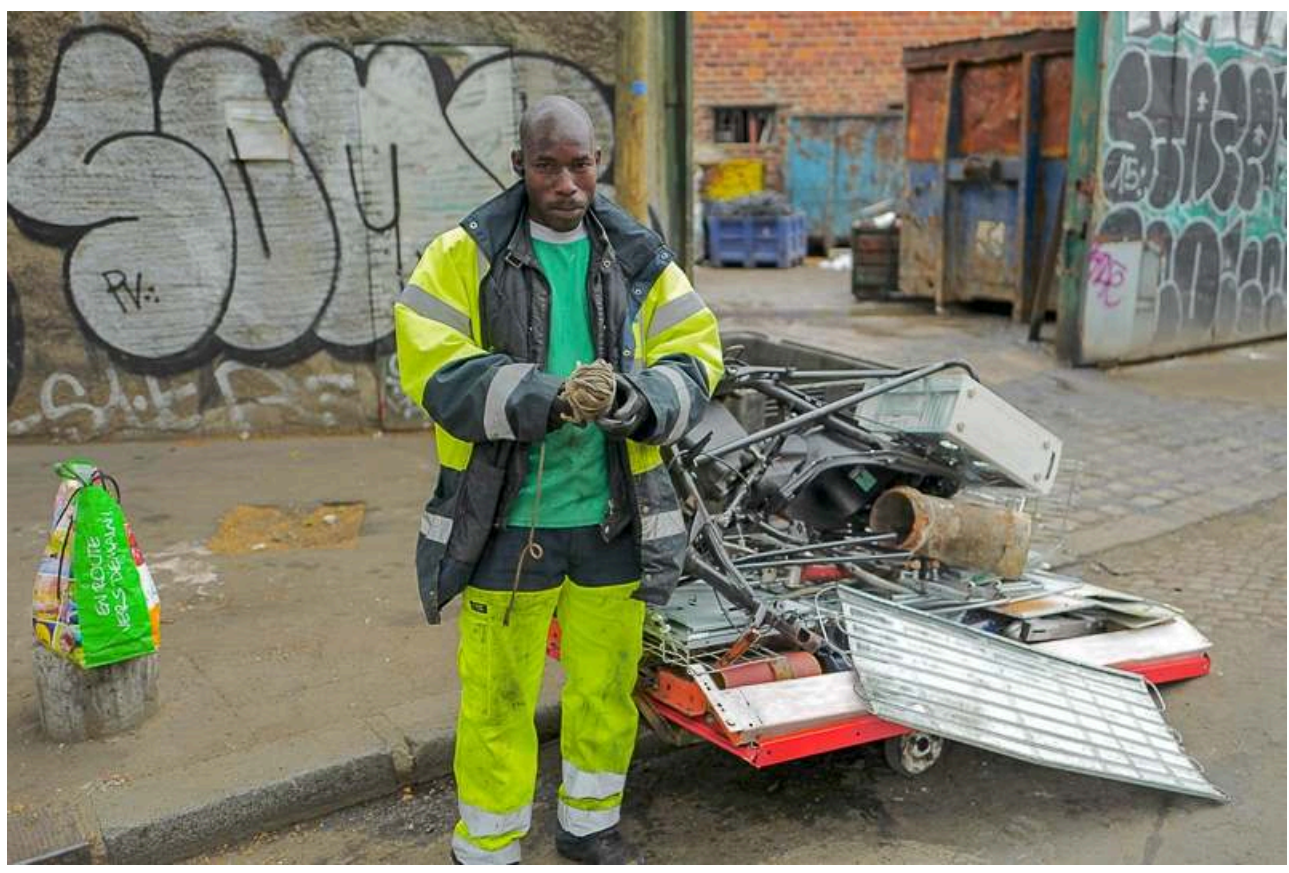

Auteur : P. Garret, juin 2016.

Plus souvent, l'entrée dans la ferraille se réalise grâce aux proches qui ont davantage d'expérience et acceptent de montrer les ficelles du métier ou de faire travailler un(e) membre de la famille nouvellement arrivé(e). C'est le cas notamment pour les Roms originaires de Roumanie rencontrés à Ivry-sur-Seine et à Saint-Denis pour lesquels les réseaux familiaux et de voisinage jouent un rôle important : les matériaux se collectent en famille, le travail de démontage ou le portage des encombrants lourds (machine à laver, réfrigérateur, etc.) peut nécessiter l'aide des voisins.

10 Aucune des personnes rencontrées ne pratiquait cette activité avant d'arriver dans la banlieue parisienne ${ }^{10}$. Viorel, qui collecte la ferraille avec son fils en banlieue sud-est, appartient à la communauté rudar ${ }^{11}$ traditionnellement attachée au travail du bois (bûcherons, menuisiers, etc.) en Roumanie. Avant d'arriver en 2008 dans le bidonville 
de la rue Truillot, à Ivry-sur-Seine, Viorel réside quelques années à Valencia, en Espagne, où vivent des parents proches l'initiant à la ferraille. Mais, à Valencia, il est embauché à récolter l'ail et les oranges, puis dans le bâtiment, la ferraille ne suffisant pas à faire vivre la famille. Dans le «bidonville Truillot », de nombreuses familles font la ferraille, comme Viorel : "J'ai commencé avec un chariot Carrefour. On cherchait dans les poubelles, dans tous les secteurs de la ville, par exemple les fers à repasser, les fours, les fils électriques. Dans le bidonville, on stockait et quand on avait $500 \mathrm{~kg}$, on appelait quelqu'un qui venait chercher tout ça en camionnette. Au bout de deux ans et demi, j'ai pu acheter une camionnette d'occasion et maintenant je fais les encombrants (...). C'est crevant, on est fatigués de la ferraille, mais qu'est-ce que je peux faire d'autre sans travail ? » (BF, Ivry-sur-Seine, 26/05/2016).

11 Simon, également Rudar, explique : "Mon beau-père était déjà ici et j'ai travaillé pour lui de 2009 à 2012, au ramassage des encombrants. J'avais un chariot pour remplir son camion et lui conduisait. Si je lui demandais son camion pour travailler pour mon compte, il me demandait 100 euros pour chaque trajet et c'était beaucoup trop cher (...). Les relations avec la famille sont difficiles et chacun travaille pour soi. Au début, on regarde comment les autres font; c'est comme ça que vient l'expérience. Mais c'est difficile, il y a beaucoup de compétition entre nous. Si tu vas tous les jours pour ramasser, tu vois tous les autres tourner tout le temps» (BF, Ivry-sur-Seine, 26/05/2016). Pour Simon, la présence de la belle-famille est d'une grande aide lors de son arrivée en France et, grâce à son beau-père, il construit son expérience dans la récupération - savoir où, comment et quoi collecter, comment trier, nettoyer et à qui revendre. Mais, dès qu'il l'a pu, et parce que les relations familiales se sont tendues, il a cherché à s'émanciper. Il y est parvenu professionnellement, en économisant pour s'acheter un véhicule, mais n'a pas réussi pour son logement qu'il sous-loue à son beaupère dans un immeuble pourtant prêté par la municipalité. Dans le domaine du travail, on retrouve des modalités d'insertion observées par ailleurs dans celui de l'habitat ${ }^{12}$. Certes, la famille élargie, la communauté, le voisinage peuvent jouer un rôle au moment de l'arrivée, de l'accès à l'habitat dans le bidonville ou, ici, de l'entrée dans l'activité de récupération, mais ce rôle est inégal, souvent ponctuel, à géométrie variable au gré des déplacements, et peut aussi générer des tensions.

Toutefois, comme nous le verrons plus loin, en dépit de cette entraide ponctuelle, les récupérateurs aspirent à prendre leur indépendance et préfèrent travailler « chacun pour soi ». 
Illustration 2 - Un travail par défaut?

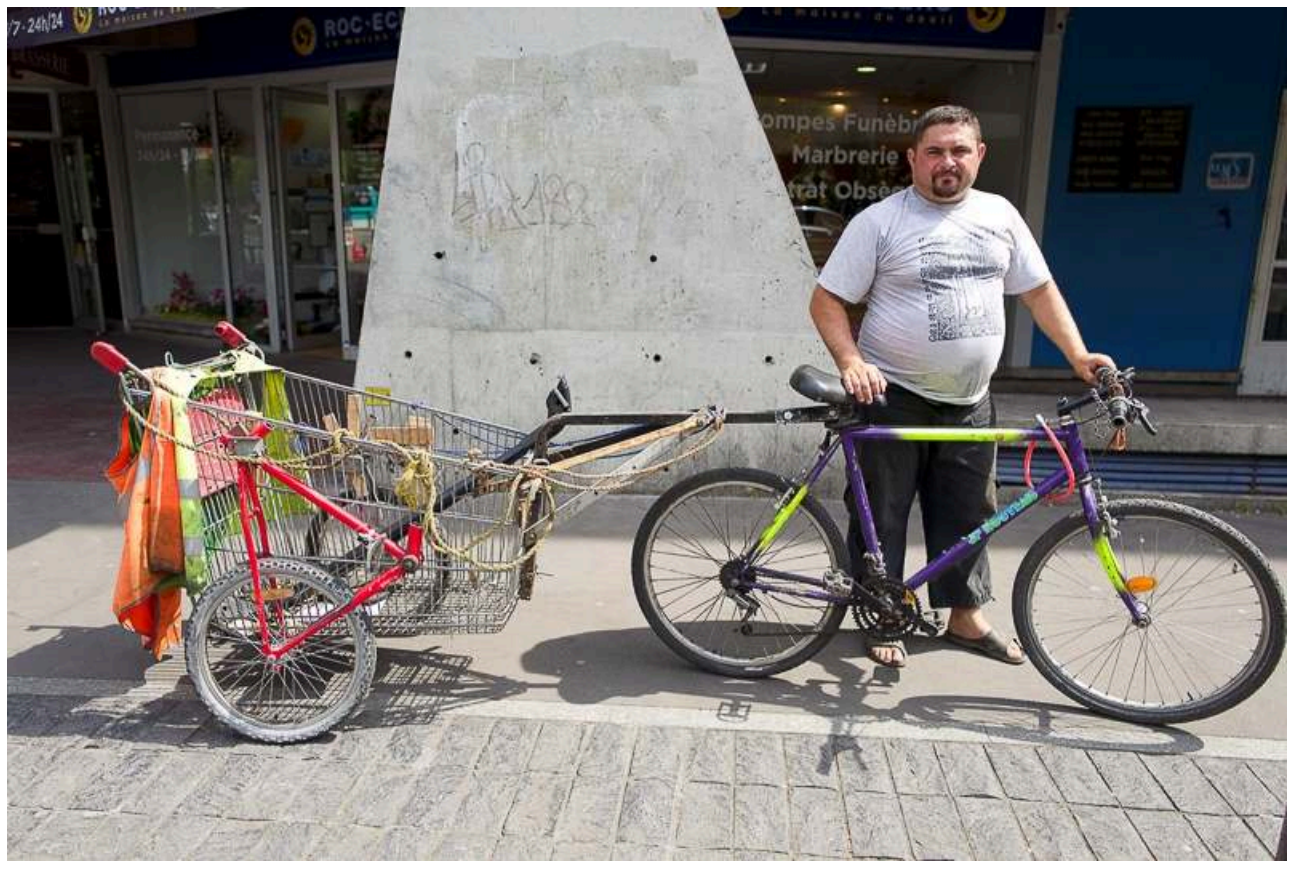

Rom roumain, Andreï relogé par la municipalité, n'a plus le droit de faire la ferraille, mais, sans ressources suffisantes, il continue pourtant son activité de collecte.

Auteur : P. Garret, mai 2017

\section{Un boulot par nécessité : une économie de la pauvreté}

Les propos des récupérateurs de matériaux étonnent parfois par leurs convergences lorsqu'ils sont tenus en des lieux très éloignés les uns des autres. À Istanbul, ceux-ci décrivent leur activité comme un vrai travail permettant d'échapper à la misère, en dépit des stigmates accolés à ce «sale boulot» (Hugues, 1996). Il s'agit peut-être du "dernier des métiers", mais néanmoins d'un métier qui évite de "tomber dans la criminalité » (Florin, 2016). En des termes presque similaires, Mona, habitant le site de relogement municipal surnommé camp Voltaire, nous explique: "Qu'est-ce que je ferais sans la ferraille? Je fais quoi sans ça? Je vole ou je mendie ? ${ }^{13}$ (BF, Ivry-surSeine, 27/06/2016).

Les activités de mendicité, biffe et ferraille sont ainsi hiérarchisées, ce qui permet de distinguer l'économie de la misère (la mendicité, la biffe) qui autorise peu l'épargne, de l'économie de la pauvreté (la ferraille) qui favorise davantage l'épargne et la projection dans l'avenir (Lasida, 2009 ; Moreau de Bellaing, 2000). Ainsi, Marco, Rom de Roumanie a commencé à son arrivée en 2002 en banlieue parisienne à « faire le déchet » (la biffe dans les poubelles et la revente aux puces). Assez rapidement, Marco se reconvertit dans la ferraille « qui paye plus », en collectant au moyen d'un chariot de supermarché. En 2009, Marco ramène de Roumanie une fourgonnette d'occasion qui permet d'améliorer ses revenus et précise: «je ramassais, je ne volais pas!». La famille se déplace de bidonville en bidonville dans les banlieues parisiennes jusqu'à son relogement en 2014. Théoriquement, il lui est interdit de continuer la ferraille, mais les aides perçues permettent à peine à la famille de subsister, d'autant que Marco a en charge ses parents âgés - qui dorment dans la fourgonnette, leur hébergement dans le 
chalet étant interdit. La journée, la fourgonnette est utilisée pour faire la ferraille en famille et, la nuit, elle est réaménagée sommairement en couchage (BF et PG, Ivry-surSeine, 28/06/2016 et 29/11/2016).

Au-delà des situations exposées ci-dessus, ces parcours montrent une évolution en deux temps: le premier est nécessaire pour passer de la biffe à la ferraille, cette dernière étant effectuée au moyen basique d'une poussette, d'un chariot de supermarché ou d'une charrette-vélo bricolée. Le second temps, lié aux capacités d'épargne, est celui de l'acquisition d'une camionnette qui rentabilisera mieux l'activité. Ces temporalités dépendent donc du capital économique, mais aussi social et relationnel. Par exemple, les personnes originaires de Roumanie maitrisant mal la langue française sont souvent dépendantes de celles venues avant, maîtrisant mieux la langue, la ville et les modalités de l'échange commercial et monétaire lié à la ferraille (Olivera, 2015, p. 504). L'isolement et le dénuement de certaines familles semblent les contraindre à rester dans la biffe alors que d'autres bénéficiant d'appuis familiaux passent plus rapidement à la ferraille. D'une certaine manière, ce processus renvoie au temps de l'insertion, mais n'est pas toujours lié aux conditions de logement: on peut déménager du bidonville au relogement et continuer la biffe ou, au contraire, passer à la ferraille, ce qui est par exemple le cas de nombreux Roms du camp Voltaire. Et, bien sûr, faire la ferraille favorise aussi les trouvailles de la biffe comme l'explique la femme de Viorel qui l'aide dans ses tournées et a découvert un grand sac rempli de vêtements propres, posé par un habitant à côté de la poubelle (BF, Ivry-sur-Seine, 26/05/2016).

Les parcours montrent aussi que c'est l'impossibilité d'accéder à un emploi salarié qui maintient ces personnes dans l'activité de ferraille: soit parce qu'elles n'ont pas le droit de travailler, soit parce qu'elles ne trouvent pas d'emploi comme l'explique Gary : «En France, il n'y a pas de travail et encore moins pour les Roms » (BF \& PG, SaintDenis, 30/05/2017). Cette assertion est avérée dans de nombreux cas, mais pourtant certains hommes quittent la ferraille pour un emploi salarié, puis y retournent parce que leur contrat s'achève ou par choix, parce que les cours des matériaux remontent et que l'on peut gagner davantage avec la ferraille qu'avec un contrat aidé. C'est ce que décide Nicolae : à la suite de son relogement par la mairie d'Ivry, il bénéficie d'un contrat aidé chez Paprec (société de recyclage), puis se fait embaucher par un Français pour des petits travaux, puis retourne à la ferraille: «Le truc pour entrer ici [être relogé], c'était de ne pas faire la ferraille, c'est pour ça que j'ai trouvé d'autres boulots. Mais ça gagne moins. Alors, sans la ferraille, comment je fais pour vivre? » (BF \& PG, Ivry-sur-Seine, 29/11/2016). Pour d'autres, cette activité paraît leur offrir plus de liberté, notamment pour les familles qui effectuent régulièrement des allers-retours entre la France et la Roumanie.

17 Faire la ferraille peut ainsi être un choix à l'instar de ce récupérateur français qui a quitté son emploi dans le bâtiment car il estime mieux gagner sa vie dans la ferraille en étant son propre patron. Il déclare gagner en moyenne 1300 euros par mois et travaille en famille, sa femme conduisant la camionnette et son fils l'aidant lors de la collecte. 
Illustration 3 - La récupération : un métier que n'importe qui peut exercer

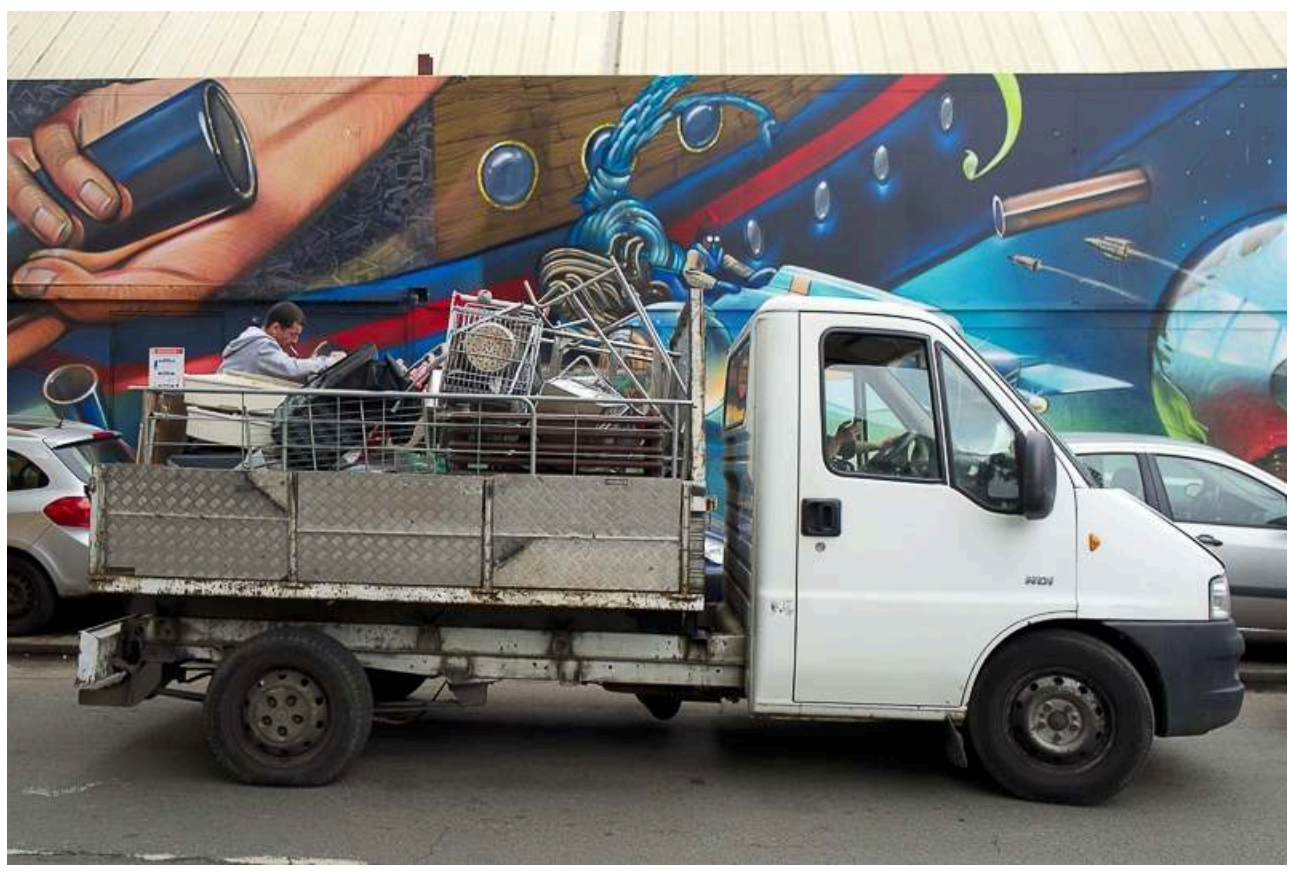

Rencontré devant le ferrailleur auquel il compte vendre sa récolte estimée à 50 euros "car c'est du mélangé, pas vraiment du lourd", ce récupérateur nous précise qu'il est français et qu'il ne veut pas être assimilé aux « Roms ». II vit « en banlieue sud, car à Paris c'est impossible, il y a que les riches qui peuvent vivre à Paris (...). Je ne gagnais pas assez dans le bâtiment pour payer mon loyer, les factures et tout pour les enfants, alors je me suis retrouvé à la rue (...). Le chariot de supermarché dans ma benne? Le ferrailleur le prendra comme le reste ! ». Un chariot en acier peut être revendu 10 euros ; considéré comme du recel, il est susceptible d'être refusé par le ferrailleur (BF \& PG, Aubervilliers, 08/06/2018).

Auteur : P. Garret, Aubervilliers, juin 2018

À cet exemple, il faut encore ajouter les ouvriers et artisans du bâtiment qui complètent leurs revenus en revendant les matériaux démontés: ces plombiers, chauffagistes, électriciens, ouvriers des entreprises de démolition, etc., stockent ces matériaux dans un garage, leur entreprise ou un coin du chantier pour atteindre la quantité suffisante justifiant le transport et la revente. Tous ces récupérateurs cherchent également des matériaux à haute valeur ajoutée (cuivre, laiton, etc.) qui occupent peu de volume et dont ils augmentent encore la valeur par le tri et démontage. Par exemple, une chaudière à gaz peut contenir entre 10 et $15 \mathrm{~kg}$ de cuivre ce qui, en fonction des cours, permet de gagner entre 45 et 70 euros alors qu'une chaudière entière, vendue au prix de la ferraille, ne rapporte pas plus d'une quinzaine d'euros. Un chauffagiste nous disait estimer cette "prime» mensuelle à 150 euros minimum.

19 Ainsi, la figure du récupérateur de ferraille ne se limite pas aux Roms pour lesquels il s'agit d'une activité relativement nouvelle. Mais ces derniers, visibles et repérables dans l'espace public, restent marqués par une sorte de double peine qui accole au qualificatif stigmatisant de rom (Lièvre, 2014) l'activité de récupération. Ils s'en défendent souvent en disant qu'« ils ne volent pas». Sans doute faut-il rappeler ici que de nombreux ferrailleurs actuels sont les descendants d'Italiens ou de Portugais qui, en leur temps, étaient aussi des migrants pauvres et stigmatisés... 


\section{Combien gagne-t-on à « faire la ferraille » ?}

Illustration 4 - La dépendance au cours des matériaux

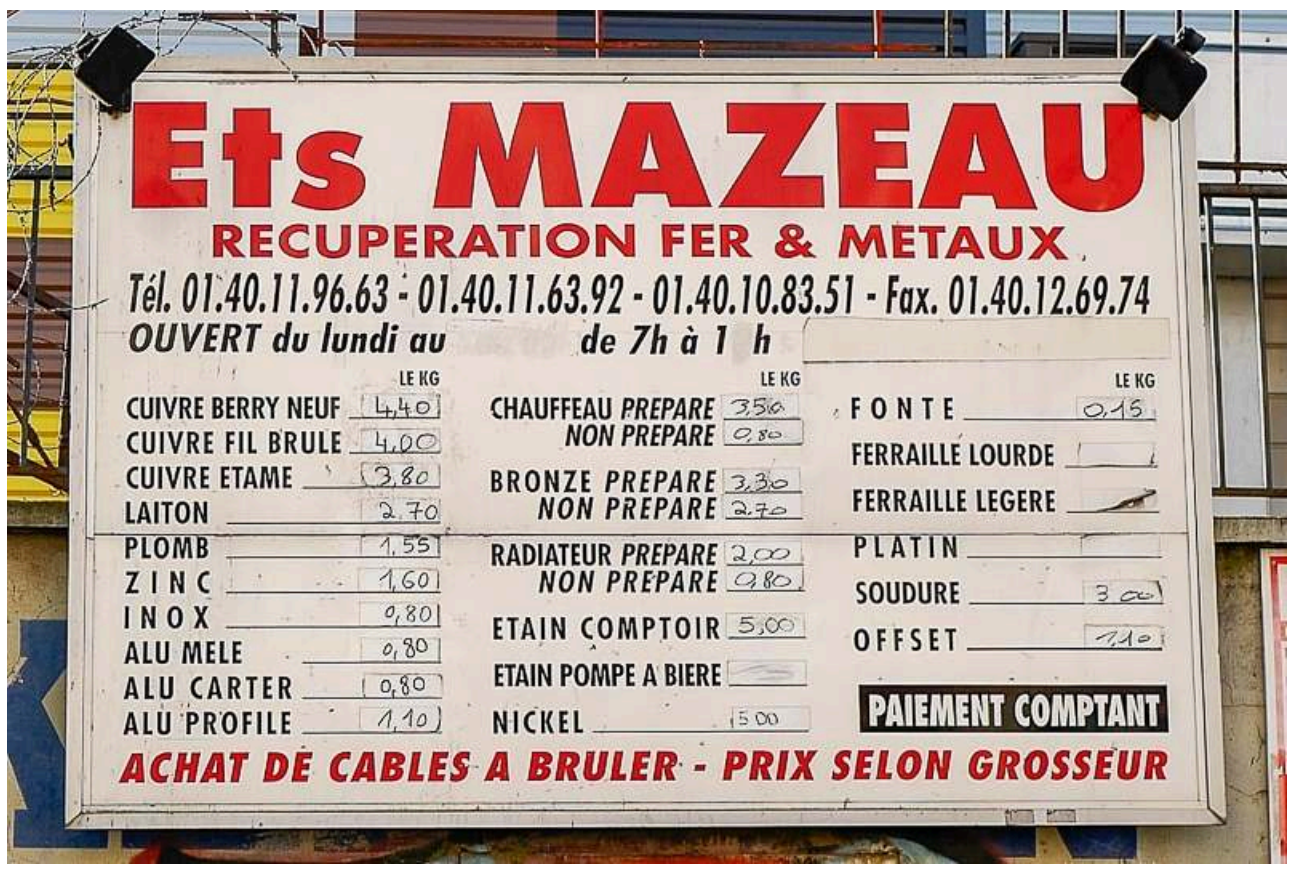

Panneau des prix des matériaux du ferrailleur Mazeau, créé en 1947 aux Puces de Saint-Ouen. Ce jour là, le cuivre est acheté 4,40 euros le kg par ce grossiste alors qu'il est coté 6,20 euros au même moment au London Metal Exchange (bourse mondiale des métaux), différence qui témoigne de la marge pour les ferrailleurs.

Auteur: P. Garret, Saint Ouen, novembre 2016.

Certes, faire la ferraille réfère à la débrouille et au bricolage, mais cette activité, bien que pénible et aléatoire, permet de mieux en vivre que d'autres, notamment pour les personnes au statut précaire et aux revenus insuffisants. Ce qu'elle rapporte dépend de la qualité des trouvailles et de la quantité collectée, liées aux moyens de locomotion, aux parcours effectués, à la connaissance des lieux de gisement, aux réseaux relationnels et au savoir-faire sur les matériaux ${ }^{14}$.

21 A priori, le fonctionnement de cette activité est formalisé par la loi 2011-900 du 30 juillet 2011 qui impose le chèque barré ou le virement bancaire pour toute transaction afin de réglementer une profession dont la majorité des paiements s'effectuaient en espèces, mais aussi afin de décourager les vols de câbles de cuivre et de matériaux sur les chantiers. ${ }^{15}$ Les objectifs de cette réglementation sont la traçabilité des matériaux et le recouvrement des impôts ; aussi, les ferrailleurs doivent-ils tenir un registre de leurs achats, y consigner l'identité et l'adresse de chaque vendeur.

À l'entrée de chaque site, une fois les matériaux pesés, souvent sous le regard attentif du patron, un «bon d'entrée » renseigne leurs qualité, poids et prix d'achat. Si la vente est supérieure à 10 euros, un chèque est établi au nom du vendeur. Si la vente est inférieure à 10 euros, le vendeur récupère un «bon de ferraille » qu'il pourra cumuler avec d'autres puis échanger le moment venu contre un chèque ( $\mathrm{PG}$, Bonneuil-surMarne, 07/06/2018). Pourtant, du côté des ferrailleurs, même les petites quantités sont intéressantes: "Les Roumains, ils nous ramènent beaucoup de bricoles à moins de 10 euros et ce n'est pas intéressant de leur faire un chèque. Mais on leur fait des bons et 
on prend tout car $1 \mathrm{~kg}$, c'est toujours $1 \mathrm{~kg}$ ! On fait juste attention à la marchandise pourrie, volée, si c'est de la merde, on n'achète pas » (BF \& PG, Saint-Ouen, 29/11/2016).

23 Ce système suppose d'avoir un compte bancaire, ce qui n'est pas le cas de tous les récupérateurs. Si Sidiki, bien que sans-papiers et squatter, a ouvert un compte à la Banque Postale, d'autres personnes sont soumises à un interdit bancaire et fichées à la Banque de France - c'est le cas de plusieurs Roms du camp Voltaire. Certains passent donc par des intermédiaires, comme l'explique Mona qui fait la ferraille avec son mari : «Parfois, on ne trouve que $300 \mathrm{~kg}$ et ça va nous faire 24 euros de bons, mais on les donne aux Français et aux Portugais contre du liquide parce qu'on n'a pas de compte et finalement, on ne va garder que 20 euros (...). Il y a des jours où on peut gagner $30 \mathrm{ou}$ 40 euros, mais d'autres jours, rien du tout, même pas de quoi payer le gasoil du camion » (BF \& PG, Saint-Denis, 27/06/2016). Ces intermédiaires prennent de $5 \%$ à $20 \%$ (parfois $30 \%$ ) des sommes gagnées, en fonction de leur proximité relationnelle avec le vendeur (Olivera, 2015, p. 105) : il s'agit de «Français », de «Portugais » mais aussi, pour les Roms, des hommes de leur propre communauté. Tout ceci fait écho à ce qu'explique Lucian : «C'est difficile la ferraille, parce que c'est lourd. Et si tu ramasses pendant deux jours pour 1 tonne que tu vas vendre à 80 euros, que tu enlèves 30 euros de gasoil, les sandwichs et le pourcentage qu'on te prend pour racheter tes bons [environ 20 euros], il ne te reste plus grand chose...» (BF \& PG, Ivry-sur-Seine, 27/07/2017). Certes, Sidiki, le Malien, travaille en indépendant, mais il n'en est pas de même pour certains Roms qui se trouvent sous la tutelle d'autres hommes bénéficiant d'une position dominante. Roxana, responsable d'un site de relogement, expliquait ainsi que les récupérateurs étaient obligés de vendre leurs matériaux à un ancien chef de bidonville qui venait régulièrement avec son camion sur place et préemptait leurs matériaux en retenant un pourcentage pour lui-même (BF \& PG, Ivry-sur-Seine, 27/07/2017).

24 L'une des façons de s'émanciper de cette dépendance est d'accéder au statut d'autoentrepreneur. Les rentrées d'argent deviennent alors justifiables et cela permet de prouver que l'on travaille légalement en cas d'arrestation par la police, même si récupérer des matériaux abandonnés ou encombrants sur la voie publique n'a rien d'illégal. Mais, ici encore, les démarches pour obtenir ce statut sont difficiles. Dans le camp Voltaire, Gheorghe, qui maîtrise le français, demande 50 euros pour tout contrat qu'il remplit. Le statut d'auto-entrepreneur, envié par beaucoup, ne permet cependant pas l'enrichissement espéré ainsi que l'explique un récupérateur «français »: «Je me fais entre 50 et 60 euros par jour, à peu près 1300 euros par mois en moyenne. La loi dit qu'ils doivent payer par chèque, et ben c'est pas vrai, il $\mathrm{y}$ en a beaucoup où on peut encore se faire payer en espèces, même chez des gros ferrailleurs (...). Moi je suis en auto-entreprise, mais je me fais payer un peu en chèque et un peu en espèces. Tu te mets deux ans en auto-entrepreneur et après il faut arrêter, sinon tu te fais contrôler... et puis tu recommences " (BF \& PG, Aubervilliers, 08/06/2018). Les contournements sont présentés comme indispensables pour s'en sortir financièrement: suspendre le statut d'auto-entrepreneur ou l'obtenir pour un autre membre de la famille, ne pas tout déclarer ou se faire payer en liquide comme l'explique un ferrailleur qui a pignon sur rue : « Est-ce que tout le monde fait des chèques?! Vous n'avez pas remarqué qu'il y a souvent des baraques à frites devant les ferrailleurs, avec des gens qui attendent? Vous pensez qu'ils sont là pourquoi? Pour changer les bons en espèces contre un 
pourcentage. (...) La ferraille, c'est comme la politique, faut que ça sente la merde, mais pas trop... » (BF \& PG, Gennevilliers, 06/06/2018).

Enfin, la variation des cours des matériaux joue un rôle sur les revenus, même si les effets peuvent en être atténués par le stockage des matériaux trouvés, mais encore faut-il que ces derniers soient achetés par les ferrailleurs au juste prix. Or, souvent, les Roms subissent une différence de traitement par les grossistes. Si être ferrailleur peut être lucratif, rester ferraillou ne vaut pas toujours opulence : Sidiki gagne en moyenne 700 euros par mois, d'autres atteignent le double ou sans doute un peu plus du fait de leurs pratiques de contournement de la législation, d'autres encore davantage lorsqu'ils possèdent plusieurs fourgonnettes et qu'ils emploient des récupérateurs « au noir » qui collectent à leur place.

Illustration 5 - Vendre au meilleur prix...

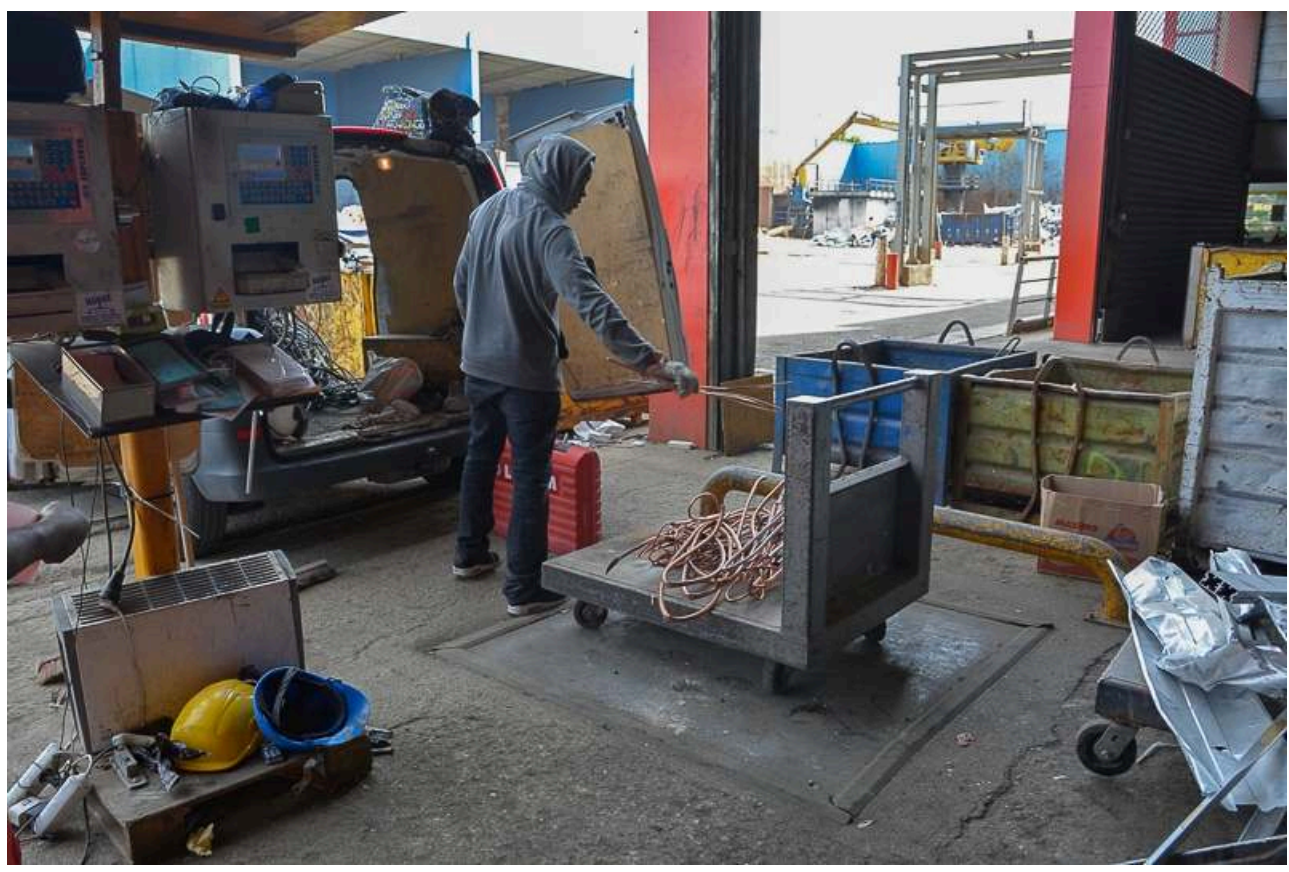

En juin 2018, un particulier pèse le cuivre qu'il vient vendre chez ce grossiste de Bonneuil-sur-Marne. II existe de légères différences de prix entre les ferrailleurs ainsi que l'explique Viorel : « On va vendre à celui qui paye le plus. Je vais chez le grossiste portugais, mais pas chez celui d'à côté car c'est un voleur ». En réalité, plus que le prix, c'est la relation établie entre ferraillou et ferrailleur qui détermine le choix de ce dernier.

Auteur : P. Garret, Bonneuil-sur-Marne, juin 2018

\section{Pratiques professionnelles : circuler, glaner, bricoler, contourner et transgresser}

La dimension spatiale des pratiques liées à la ferraille est essentielle puisque la ville constitue pour ces «braconniers $»^{16}$ que sont les ferraillous une ressource au sens propre et, notamment, ses marges qu'ils exploitent pour leurs activités. 


\section{La ville comme ressource : circulations et temporalités du glanage}

Arrivé dans un bidonville d'Ivry en 2008, Viorel, après avoir commencé la récupération avec un chariot de supermarché, s'achète une fourgonnette d'occasion immatriculée en Roumanie: "Maintenant, j'ai un Renault Master, je connais les jours de sortie des encombrants et j'ai la liste par internet pour toutes les villes (...) on vit mieux qu'avant, merci à Dieu!». Simon, son voisin, complète: «Je travaille dans la ferraille de $7 \mathrm{~h}$ à $23 \mathrm{~h}$ presque tous les jours (...). Quand on revient ici, on a une petite place près de la maison pour faire le tri et démonter le cuivre et ça pose des problèmes avec les voisins. Quand on trouve un appareil qui marche, on le revend et ça c'est mieux, sinon on revend au ferrailleur portugais » (BF, Ivry-sur-Seine, 26/05/2016). À la différence de ceux qui collectent au moyen d'un vélo bricolé et qui pratiquent une maraude fatigante et peu lucrative, les propriétaires de fourgonnettes rentabilisent mieux leur activité : beaucoup, comme Mona et son mari, utilisent un GPS et élaborent une cartographie logique de leur parcours en fonction des sorties des encombrants de manière à dépenser le moins d'essence possible, même si ces collectes peuvent parfois les conduire à $200 \mathrm{~km}$ de Paris. Le principe est de passer avant la collecte officielle, ce qui n'est pas toujours possible.

Illustration 6 - Le jeune homme à la bicyclette

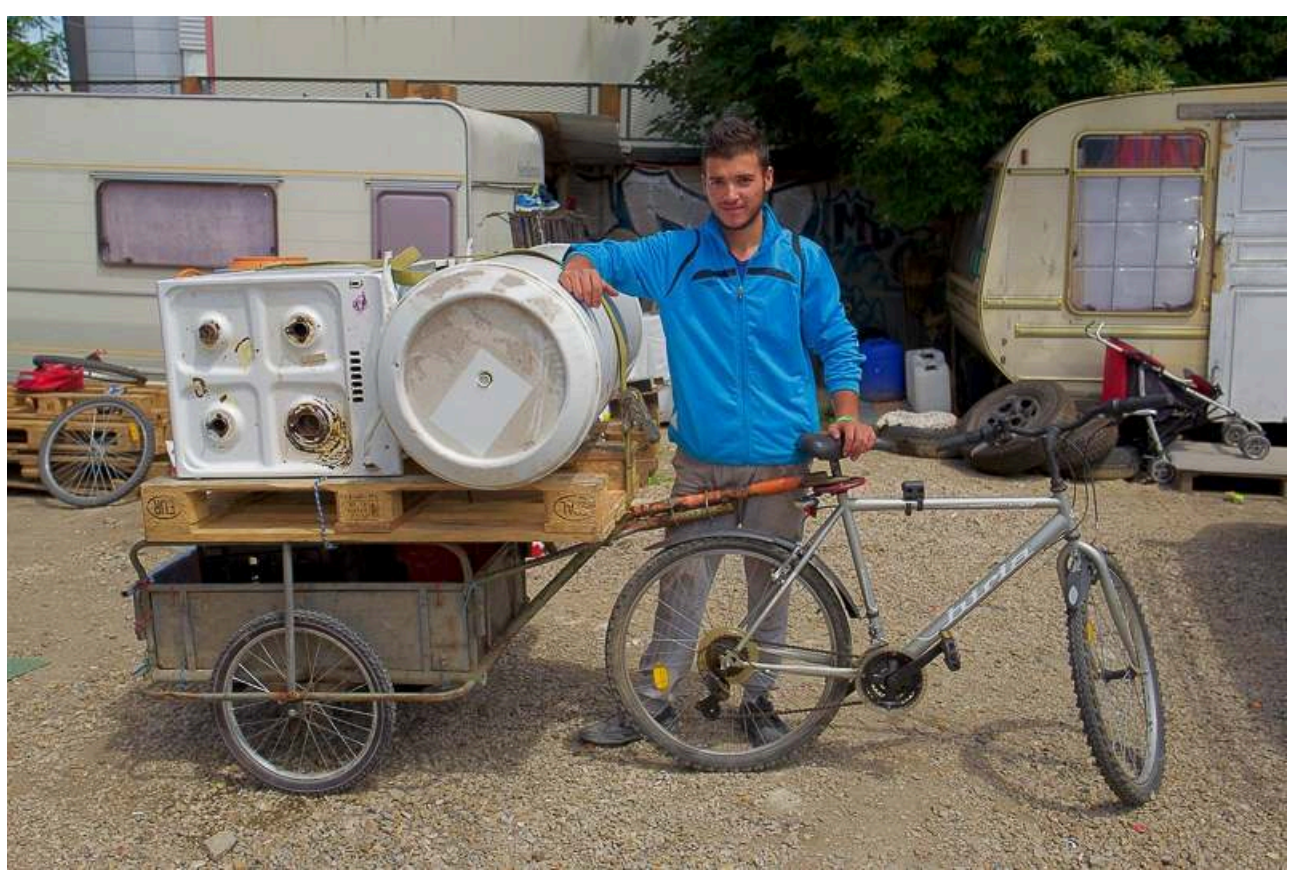

À Ivry-sur-Seine, l'association Convivances a négocié l'occupation d'une friche urbaine pour des familles Roms. Celles-ci ont une activité liée à la ferraille, autorisée par l'association, au moyen de fourgonnettes ou d'un vélo et d'une carriole comme ce jeune homme.

Auteur : P. Garret, Ivry-sur-Seine, juin 2017.

Sidiki, pour sa part, s'est bricolé un plateau d'1,50 $\mathrm{m}$ de long sur $50 \mathrm{~cm}$ de large, auquel il a fait souder deux roues et un manche. Il chemine à pied et pousse son attelage devant lui, sans pouvoir emprunter les trottoirs. Si son chariot lui permet de porter de lourdes charges, il le contraint à des horaires nocturnes afin d'échapper à la circulation. Aussi, part-il à 2 ou $3 \mathrm{~h}$ du matin pour trois tournées entrecoupées de passages chez le ferrailleur ou à son garage, lieu de stockage, totalisant entre 15 et 
$20 \mathrm{~km}$ par jour. À midi, il rentre chez lui se reposer. De confession musulmane, le seul jour de congé qu'il s'octroie est le vendredi.

Illustration 7 - Les longs trajets de Sidiki

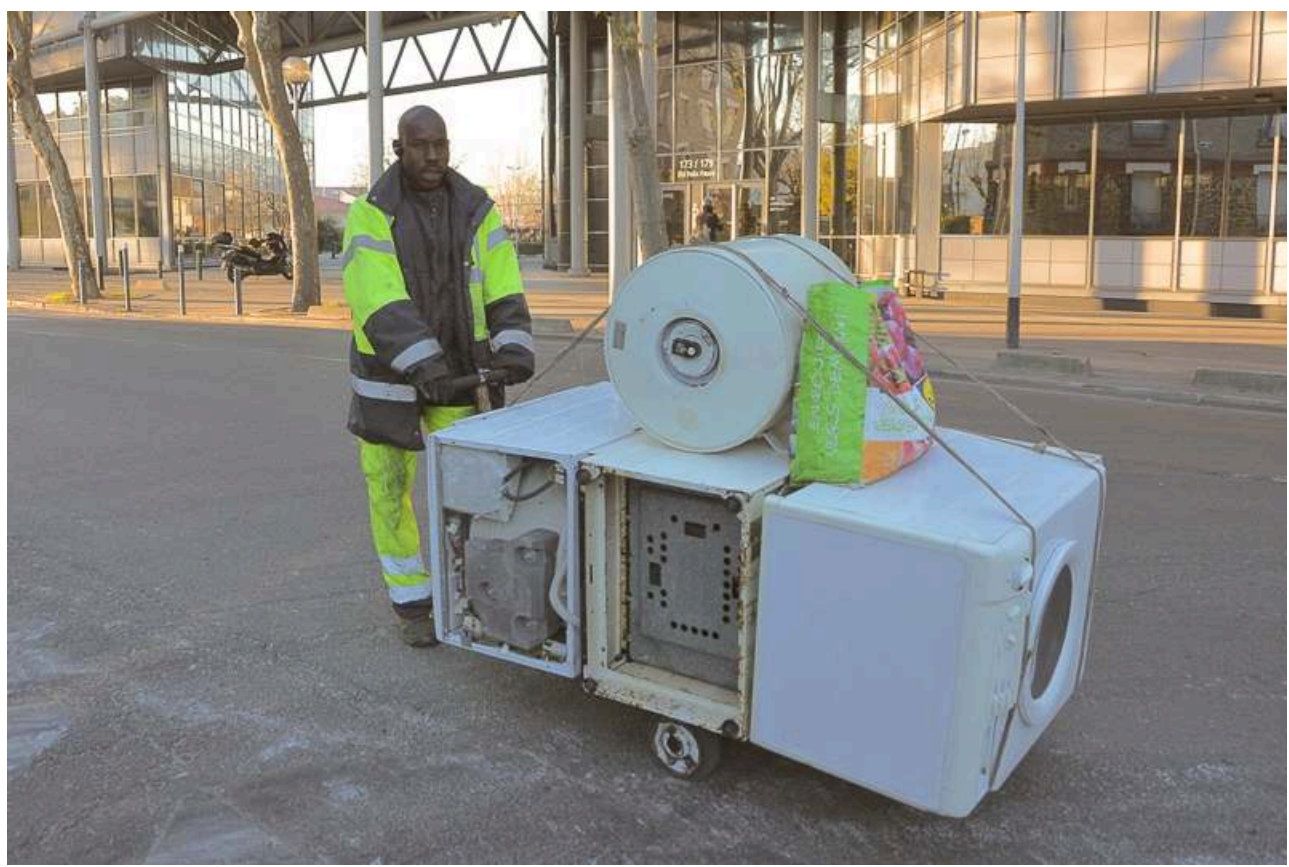

L'attelage de Sidiki est ici chargé de trois lave-linge et d'un chauffe-eau. Sa façon de collecter a évolué au début, il maraudait, mais, de plus en plus, des amis maliens l'appellent pour lui indiquer des gisements via son « oreillette bluetooth ». La tactique adoptée par Sidiki le rend visible lorsqu'il circule il revêt un costume fluo semblable à celui des agents d'entretien. II explique n'avoir jamais été interpellé par la police. Lorsqu'il trouve de l'électroménager en état de marche, il le revend à un compatriote qui l'emmène par camion en Afrique. II récupère des morceaux de bicyclette qu'il reconstitue en vélos en état de rouler qu'il revend également.

Auteur : P. Garret, novembre 2016.

Aux temporalités spécifiques des tournées s'ajoute la connaissance des lieux de gisement de la ressource qui s'affine avec l'expérience et une forme de culture de la ville - « Nous connaissons mieux Paris que vous! » dit Mona. Surtout, il apparait que, progressivement, les récupérateurs constituent des réseaux de relations qui les aident à localiser les ressources. C'est le cas pour Sidiki, mais aussi pour Mona ou pour Çiprian, son voisin, expliquant qu'ils connaissent beaucoup de gens qui les appellent pour débarrasser des objets volumineux ou des gravats. Arrivé en 2005 en France, Çiprian a fait la manche pendant trois ans, puis s'est mis à la ferraille avec son père et son frère, d'abord au moyen d'un chariot de supermarché, d'un vélo et, enfin, d'un Renault Traffic d'occasion. Aujourd'hui, Çiprian calcule la distance à parcourir et la rentabilité de ce qu'on lui propose avant d'accepter: "Si c'est à Paris ou près d'ici, j'y vais. Aujourd'hui, on m'a appelé pour de la ferraille, mais je n'y vais pas parce que je suis fatigué et parce que c'est loin (...). Le problème, c'est que les magasins comme Darty reprennent les vieilles machines. On peut en trouver, mais c'est pas facile !». Çiprian a connu quelques déboires lors de ses collectes : une gazinière, ramenée dans sa maison en Roumanie, était remplie de cafards ; une « collecte» sur un chantier a mal tourné : "J'ai récupéré beaucoup de cuivre et de plomb sur un chantier, mais un Noir m'a cassé la gueule. Mais c'était pas comme aujourd'hui, je ne parlais pas bien... Bon, le chef de chantier est arrivé et a dit que c'était lui qui m'avait donné tout ça ". En juin 2016, 
Çiprian n'était plus intéressé par la collecte en raison de la baisse des cours des métaux. Par contre, trouver de l'électroménager en bon état restait rentable, disait-il alors, car il pouvait l'utiliser pour lui-même, le revendre à des «Africains » ou l'emmener pour le vendre en Roumanie (BF \& PG, Saint-Denis, 27/06/2016). Ainsi que l'explique Martin Olivera, une autre façon de récupérer de l'électroménager procède "des "livraisons" opérées sur le terrain par des sous-traitants de grandes enseignes commerciales qui livrent à domicile et, ce faisant, se voient dans l'obligation de récupérer les anciens appareils (loi de 2006). En échange de quelques billets, ils se débarrassent ainsi d'objets encombrants de peu de valeur » que les Roms pourront revendre s'ils sont encore en état ou réparables (Olivera, 2015, p. 506).

Les femmes rom sont également très présentes dans l'activité de collecte: elles récupèrent des objets légers, donnent un coup de main pour porter et gardent la fourgonnette. Dans certains cas, elles parlent mieux français que leur mari et se chargent de la vente chez le ferrailleur; elles peuvent aussi être détentrices du statut d'auto-entrepreneur et bénéficier d'une certaine indépendance, notamment financière ${ }^{17}$.

31 Pour les plus expérimentés, les façons de circuler en ville, les rythmes de l'activité et la rentabilité de celle-ci sont liés à la possession d'un véhicule, mais aussi au «carnet d'adresses ", peu à peu constitué à l'exemple de ce récupérateur "français » dont le réseau est constitué de magasins qu'il va débarrasser sur simple demande ou, encore, à l'exemple de Mona, dont l'entregent constitue un atout indéniable ${ }^{18}$.

\section{Bricoler les « monstres » ou l'ajout de la valeur}

C'est le bricolage effectué sur les encombrants ou les «monstres »- terme employé par les artisans et ferrailleurs - qui ajoute de la valeur à la collecte : retirer le cuivre d'une chaudière ou le tambour en inox d'un lave-linge, dégainer les fils électriques, extraire les moteurs et autres composants. Ceci implique d'avoir des outils, de savoir comment procéder, de connaître les matériaux et leurs prix. Ce bricolage se réalise à même la fourgonnette en raison du manque de place ou de l'interdiction de faire la ferraille imposée dans certains sites de relogement. Alors, la discrétion est de mise et la fourgonnette se gare dans un endroit peu visible. 
Illustration 8 - Le casseur de moteurs

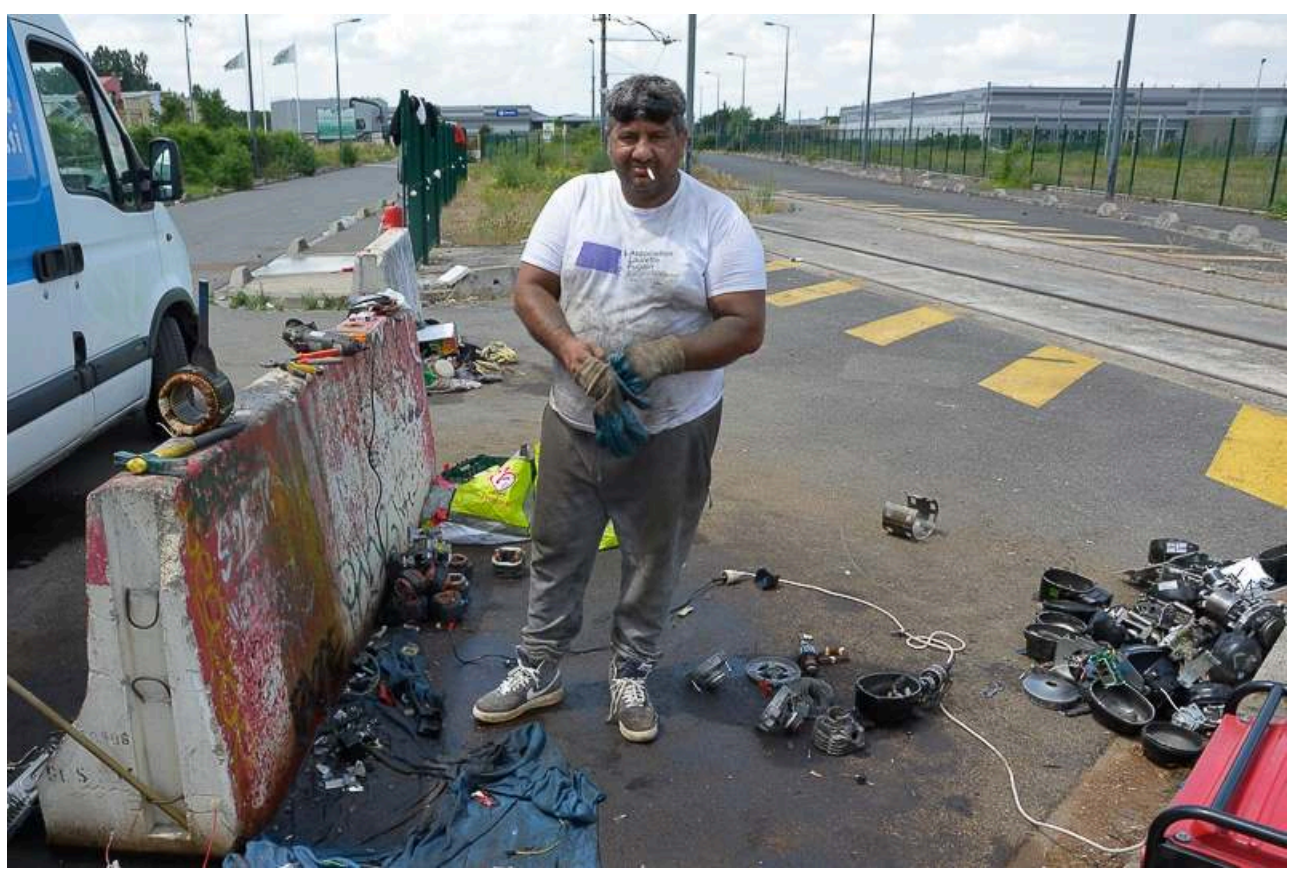

Originaire de Bulgarie, ce récupérateur rom démonte des moteurs pour en séparer les composants et extraire le cuivre. II utilise une petite tronçonneuse alimentée par un groupe électrogène. Son rythme se divise en deux journées de glanage suivies d'une journée de démontage dans ce lieu discret, proche d'un ferrailleur. Il collecte avec sa femme qui, ici, nettoie la camionnette et fait la lessive grâce à la borne incendie que l'on entraperçoit sous le grillage où sèche le linge.

Auteur : P. Garret, Bonneuil-sur-Marne, juin 2018. 
Illustration 9 - Le « camp » de l'avenue Pierre Semard à Ivry-sur-Seine

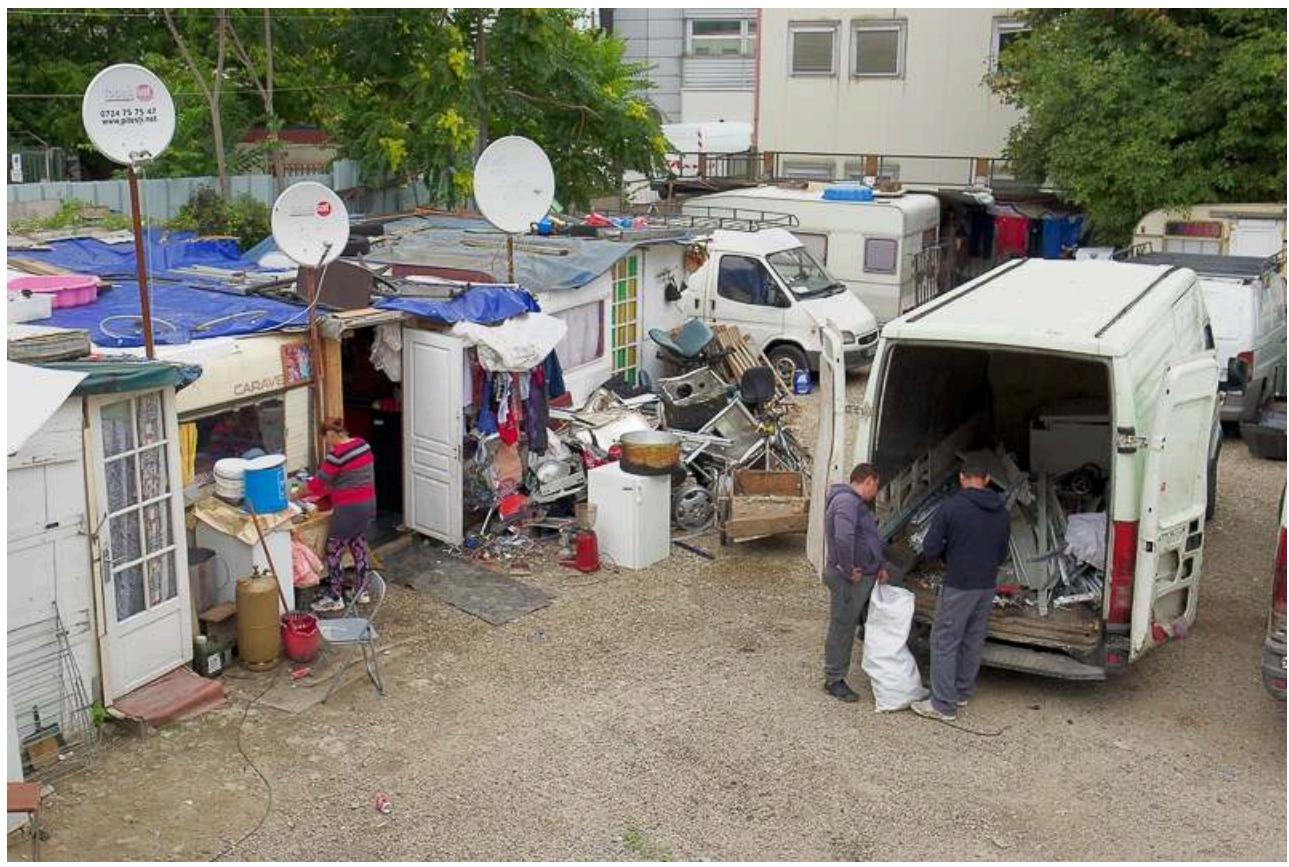

Avant de vendre au ferrailleur, il faut trier, démonter et parfois stocker. Mais accumuler la ferraille reste mal perçu des riverains et des autorités. Les résidus invendables finissent parfois dans les conteneurs du quartier ou sur les trottoirs, suscitant le mécontentement de la mairie. Ceci explique l'interdiction de faire la ferraille, fréquente dans les sites de relogement : ainsi, certains Roms du bidonville Truillot ont préféré refuser le relogement et partir ailleurs pour continuer leur activité de récupération (BF \& PG, Ivry-sur-Seine, 31/10/2017).

Auteur : P. Garret, Ivry-sur-Seine, septembre 2017.

Quant aux plombiers, chauffagistes et électriciens, ils s'intéressent essentiellement aux matériaux de valeur, délaissant, par exemple, les ballons d'eau chaude peu rentables. Mais, parfois, plutôt que de les apporter à la déchetterie, « on les laisse sur le trottoir et ils disparaissent aussitôt ", récupérés par d'autres qui y trouveront leur intérêt. Pour ces artisans, le temps du bricolage reste un critère déterminant la valeur de la trouvaille: "Sur une chaudière à gaz, tu as besoin d'une heure de boulot pour démonter, c'est pas difficile et rentable, mais par exemple pour séparer le cuivre du laiton, ça demande plus de temps et il faut y aller au chalumeau. (...) Il faut être très patient pour ce boulot et, moi, j'ai jamais vu un plombier s'enrichir grâce à ça, mais ça fait une petite prime !» (BF \& PG, Saint Ouen, 27/07/2018).

\section{Contourner, transgresser et la construction d'une catégorie spécifique de ferraillous : les Roms}

La ferraille constitue un petit monde au sein duquel presque tous se connaissent et où tout se sait rapidement. Hormis les occasionnels, les ferraillous sont souvent fidèles à leur grossiste et les ferrailleurs, quant à eux, sont reliés par des échanges commerciaux mais aussi par des liens établis depuis longtemps en raison du caractère familial de leurs entreprises. En quête de reconnaissance, les ferrailleurs tendent à mettre en valeur leur contribution à la préservation de l'environnement et leurs bonnes pratiques, mais, du côté des ferraillous, la suspicion de "marchandise sale » à la provenance douteuse reste de mise et contribue à construire une figure spécifique 
négative. Elle est même marquée par une sorte de double peine lorsqu'il s'agit du Rom récupérateur de ferraille. Ainsi, les Roms ne semblent jamais être des ferraillous comme les autres, mais forment une catégorie élaborée sur les représentations, discours et pratiques des autres acteurs ${ }^{19}$. L'un des ferrailleurs proche du site de relogement Voltaire - qu'il qualifie de «bidonville» - nous certifie ne rien acheter aux Roms qui y vivent, même si l'on voit ceux-ci attendre devant son entreprise : « Je refuse ceux-là du bidonville, avec eux il y a des problèmes et on ne sait pas d'où vient la marchandise (...). Les autres particuliers, oui ça va, parce que je les connais, c'est $\mathrm{OK}$, mais pas les Roms » (BF \& PG, La Courneuve, 08/06/2018). Parfois, les Roms - facilement assimilés aux gens du voyage - ne peuvent négocier des tarifs qui les désavantagent de façon évidente (Bernardot et al., 2016) : "Les gens du voyage ou les Roms, on leur achète au plus bas prix car ils bloquent l'entrée et avec eux t'as plus de risques d'emmerdes (...) s'ils nous amènent des trucs louches, on n'en veut pas, on ferme la porte (...). Quand on ne veut pas trop de Roms, on ne fait pas dans le détail de ce qu'ils ont dans leur camionnette et on sous-évalue le prix, comme ça on est tranquille » - ce qui augmente aussi la marge du ferrailleur... (BF \& PG, Gennevilliers, 06/06/2018). À Bonneuil, un autre patron explique : «J'ai beaucoup de Roms, ils marchent par familles et ils viennent avec 3 ou 4 camionnettes, mais il y en a qu'un seul qui a un compte en banque. C'est difficile à gérer à cause de la sécurité et de leurs gosses qui courent dans la ferraille, alors je tiens pas à trop en avoir; ça se joue sur les prix » (BF \& PG, Gennevilliers, 06/06/2018).

Pourtant, les récupérateurs, qu'ils soient ou non Roms, connaissent assez bien la valeur de leur chargement. Selon Gheorghe, «Les balances des ferrailleurs sont trafiquées et les gitans perdent 100 ou $200 \mathrm{~kg}$ mais ils n'osent rien dire ». (BF \& PG, Saint-Denis, 28/11/2016). Les Roms rencontrés sont tout à fait conscients du stigmate qui les marque. Ils se défendent de collecter de la "marchandise sale ", notamment parce qu'ils savent qu'elle pourra être refusée par le ferrailleur. Certes, ceci n'empêche pas des façons de faire douteuses, mais il s'agit souvent de personnes débutant dans l'activité : en 2016, un couple Rom, ne parlant pas du tout français et visiblement très démuni, apportait chez un ferrailleur de la Plaine Saint-Denis, un chariot Leroy-Merlin chargé d'une grande plaque de chantier sur laquelle était posée une plaque d'égout en fonte et trois bouteilles de Butagaz. Ce type de situation n'est pas l'unique fait des Roms et les observations à l'entrée des ferrailleurs montrent assez rapidement que des particuliers non roms apportent aussi des objets à la provenance douteuse...

Pourtant, les non Roms distinguent leurs pratiques de celles des Roms, à l'instar de ce « Français » expliquant qu'il ne travaille pas comme les Roms alors que, à l'évidence, son camion apporte le même type de matériaux... Ou encore, un chauffagiste assez virulent à l'égard des Roms, amalgamés aux gens du voyage : « Tout disparaît, même les entourages d'arbres, les plaques d'égouts car la fonte, c'est du lourd, ça se vend bien. Les gens du voyage, ils sont malins, tu ne sais pas d'où ça vient. Par exemple, ils amènent des bobines de cuivre toutes neuves, tu sais que c'est volé, mais le ferrailleur s'en fout. Il va les prendre et vite les expédier ailleurs pour que ça ne reste pas trop longtemps et ne pas avoir de problème. Il y en a même qui vont piquer à la déchetterie et on les laisse faire car ça arrange tout le monde...» (BF \& PG, Aubervilliers, 07/06/2018 et Saint Ouen, 27/07/2018).

Les récupérateurs Roms, ou assimilés comme tels, disent parfois être confrontés à la police qui arrête leurs véhicules, et même leurs vélos, contrôle leurs papiers et les 
traite au mieux avec condescendance, au pire avec brutalité verbale. Leurs relations avec les habitants oscillent entre l'indifférence, le mépris mais aussi l'empathie, manifestée par des dons.

Aux yeux des institutions et de la plupart des associations, la biffe et la récupération restent des activités hors normes. Pour les Roms du camp Voltaire, faire la ferraille est toléré par l'association qui gère le site, mais tend à être empêché par la municipalité qui a interdit le stationnement de leurs camionnettes à proximité du site. À Ivry-surSeine, les Roms hébergés au Village de l'espoir sont interdits de biffe et de ferraille même lorsqu'ils n'ont pas les moyens de subvenir à leurs besoins par ailleurs et que certains continuent cette activité clandestinement. La responsable de l'une des associations propose de remettre les Roms dans "le droit commun ", ce qui rime avec "le droit chemin », car faire la ferraille reste une activité associée au bidonville.

Enfin, le discours commun renvoyant au fait que les Roms préféreraient être indépendants, travailler librement et refuser le salariat est remis en cause par les entretiens qui témoignent plutôt de la difficulté, de la pénibilité et du peu de revenus tirés de la ferraille. Beaucoup désirent obtenir un «vrai travail» et en cherchent un, souvent sans succès. Aussi, les propos sur la "liberté » qu'offrirait la ferraille nous paraissent davantage ressortir de l'auto-justification et de l'auto-valorisation que d'un vrai choix - d'autant qu'aucun d'entre eux n'était dans la ferraille avant leur migration.

En bref, chacun se situe ou est situé par l'autre à la marge du système, entre légal et illégal, formel et informel, mais les Roms restent, de plus, cantonnés dans une catégorie à part et assignés à une place spécifique dans le petit monde de la ferraille.

\section{Conclusion. Les soutiers de l'industrie du recyclage}

41 Adoptant des stratégies et des parcours de collecte différents en fonction de leurs moyens, inégaux et hiérarchisés, mais qui ont leur logique propre, les récupérateurs rencontrés ont tous une connaissance approfondie de la métropole parisienne et des lieux de gisement de la ressource, via leurs réseaux d'informateurs ou via le calendrier de sortie des encombrants. Ils en utilisent les espaces délaissés et discrets pour trier, démonter, bricoler avant d'aller revendre au ferrailleur et ce que d'autres considèrent comme des espaces à l'abandon ou à la marge (tel le bidonville) constituent pour eux de précieuses opportunités spatiales que les politiques publiques de relogement ou les grands travaux de rénovation urbaine mettent à mal.

Pour les Roms, faire la ferraille se réalise en famille ou en couple et s'appuie sur des échanges d'expériences et d'entraide qui n'empêchent pas non plus les concurrences et les positions dominantes d'intermédiaires de la communauté qui préemptent les matériaux collectés par d'autres ou qui, disposant d'un compte en banque, prennent leur dîme dans les transactions monétaires.

Par cette activité, les Roms et exilés tentent de s'affranchir de la mendicité et de la biffe et, plus largement, de leur situation marginale, faute d'un emploi salarié qui leur parait inaccessible ou qui les cantonne dans des contrats aidés, souvent liés aux déchets, au réemploi ou à un travail peu valorisé socialement (tri chez Paprec, Emmaüs, ménages ponctuels...).

Roms et exilés restent aussi toujours plus vulnérables que les autres ferraillous parce que plus visibles, plus repérables et davantage en but à des ennuis et des brimades. 
Conscients de cette vulnérabilité, ils s'y ajustent comme Sidiki qui porte un uniforme d'agent d'entretien ou comme d'autres qui disent laisser les lieux propres après leur passage pour ne pas avoir de problèmes avec les habitants et les autorités. Quoi qu'il en soit, et en dépit d'une représentation commune les associant à la ferraille (et aux caravanes), les Roms composent toujours une catégorie ethnicisée à part (Lièvre, 2016), ici construite par les discours des autres ferraillous et ferrailleurs qui, pourtant, n'ont parfois pas des pratiques bien différentes et qui s'arrangent aussi avec la législation tout en traitant les Roms de façon inégalitaire.

Il nous semble que c'est plutôt l'exclusion de ces derniers du marché du travail et, plus généralement, leur exclusion sociale qui les cantonne à une économie grise, certes parfois entachée de pratiques douteuses. Surtout, ces glaneurs, qui en échange de leur travail de délestage, tri, démontage et bricolage de quantités importantes de matériaux destinés au réemploi, ne forment-ils pas la main d'œuvre à bas coût, sans droits sociaux ni reconnaissance symbolique, d'une industrie du recyclage florissante? Au Nord, comme au Sud, ces soutiers de la ville (Florin, 2015), au travail pénible et nocif pour leur santé, participent pourtant, à leur façon, à l'économie mondialisée et très lucrative du recyclage...

\section{BIBLIOGRAPHIE}

ANR Marg-In [En ligne]. http://www.agence-nationale-recherche.fr/Projet-ANR-15-CE28-0006

Balan H., 2014. L'institutionnalisation des marchés informels à Paris : une situation d'entre-deux dans le gouvernement des espaces publics. Carnets de géographes [En ligne], $\mathrm{n}^{\circ}$ 7. http:// journals.openedition.org/cdg/387

Bernardot M., Cousin G, Lemarchand A., Mésini B., 2016. Camp et campements. Multitudes [En ligne], n64. http://www.multitudes.net/camp-et-campements/

Brivot P., 1874. Le chant patriotique du drillou, du ferraillou et du chiffonnier. Paris, Hachette BNF, $32 \mathrm{p}$.

Corteel D., Le Lay S. (dir.), 2011. Les travailleurs des déchets. Toulouse, Érès, 331 p.

Cousin G., Legros O., 2014. Gouverner par l'évacuation ? L'exemple des « campements illicites » en Seine-Saint-Denis. Annales de géographie, n 700, p. 1262-1284.

Études Tsiganes, https://www.cairn.info/revue-etudes-tsiganes.htm

Florin B., 2015. Les chiffonniers du Caire. Soutiers de la ville ou businessmen des ordures?

Ethnologie française, 2015/3, n 153, p. 487-498.

Florin B., 2016. De l'indignité à l'indignation : petites luttes, résistances quotidiennes et tentatives de mobilisation des récupérateurs de déchets à Istanbul. Cultures \& conflits [En ligne], $\mathrm{n}^{\circ}$ 101, p. 99-120. http://journals.openedition.org/conflits/19184

Hasdeu J., 2009. Les Zlatari, entre ghettoïsation de la misère et identité de "patrons". Réflexions sur l'or rom », Etudes Tsiganes, vol. 38, p. 122-143. 
Hughes E.-C., 1962. Good people and dirty work. Social Problems, vol. X [édition française : 1996. Le regard sociologique. Essais choisis, Paris, EHESS].

Lasida H., 2009. La pauvreté : une approche socio-économique. Transversalités, $n^{\circ} 111$, p. 35-47.

Legros O., Olivera M., 2014. La gouvernance métropolitaine à l'épreuve de la mobilité contrainte des Roms migrants en région parisienne. EspacesTemps.net [En ligne] Travaux, 2014. https:// www.espacestemps.net/articles/lmobilite-contrainte-des-roms-migrants-en-region-parisienne/

Lièvre M., 2014. «Ceux-là sont peu soignés, peu débrouillards ». Ethnographie des Roms roumains migrants à Montpellier. Entre enjeux moraux et appartenance sociale. Migrations Société, vol. 152, p. 103-118.

Lièvre M., 2016. Roms roumanisés, Ciurari, Ursari : ethnicité et appartenances sociales. Ethnographie des migrants roms roumains à Montpellier. Revue européenne des migrations internationales, vol. 32, p. 35-57.

Moreau de Bellaing L. 2000. Économie de la pauvreté et économie de la misère. Socio-anthropologie [En ligne], $\mathrm{n}^{\circ}$ 7. http://journals.openedition.org/socio-anthropologie/100

Milliot V., 2010. La ville informelle et le travail des marges. In Milliot V., Tastevin Y.-P., Les archipels de la Goutte d'Or. Analyse anthropologique d'une métropolisation par le bas. Programme Culture et territoires en Ile-de-France, Rapport final, p. 7-89.

Milliot V., 2011. Les biffins, ou l'organisation de la misère? Territoires, p. 46-49.

Olivera M., 2015. Insupportables pollueurs ou recycleurs de génie ? Quelques réflexions sur les « Roms » et les paradoxes de l'urbanité libérale. Ethnologie française, nº 153, p. 499-509.

Scott J.-C., 2009. La domination et les arts de la résistance. Fragments du discours subalterne. Paris, Éd. Amsterdam, 269 p.

URBAROM [En ligne]. https://urbarom.hypotheses.org/

Vitale T., Mayer N., Tiberj V., Michelat G., 2018. Le lent reflux des préjugés anti-Roms. In La lutte contre le racisme, l'antisémitisme et la xénophobie. Année 2017. Paris, La Documentation Française, p. 107-124.

\section{NOTES}

1. Au XIX ${ }^{\mathrm{e}}$ siècle, la fabrication de chapeaux à partir de peaux de lapins est un artisanat florissant, basé sur la récupération ou l'achat des peaux par les chiffonniers. https://www.francepittoresque.com/spip.php?article8200

2. http://costella.fr/

3. Les entretiens sont localisés et datés entre parenthèses. BF et PG correspondent respectivement à $\mathrm{B}$. Florin et $\mathrm{P}$. Garret.

4. https://www.derichebourg.com/fr/accueil/qui-sommes-nous/notre-histoire

5. Expression utilisée par Mme Mouket, responsable de l'insertion professionnelle à l'association Alteralia (entretien 31/05/2017).

6. Cet article est issu d'une recherche réalisée dans le cadre de l'ANR MARG-IN MARGinalisationINclusion, les effets des politiques de régulation des populations étrangères: le cas des migrants dits «Roms » dans les villes d'Europe Occidentale (sous la responsabilité d'Olivier Legros, Université de Tours, 2015-2019). Initialement, les entretiens, menés avec Nora Semmoud, portaient sur les modes d'insertion économique des Roms roumains pratiquant la récupération, puis ils se sont élargis à des récupérateurs non Roms ainsi qu'aux ferrailleurs de la région parisienne. 
7. Le terme «Rom» est une catégorie floue, englobant une grande diversité de personnes d'origines, de langues et de communautés différentes. Nous n'entrerons pas ici dans l'analyse de cette catégorie, dont la construction a donné lieu à une abondante littérature. Par contre, cette catégorie a un sens pour les acteurs non Roms qui les désignent ainsi. Voir sur ces débats : William, 1987 ; Olivera, 2015 ; Foisneau, 2016 et les livraisons de la revue Etudes Tsiganes.

8. Nous remercions vivement Grégoire Cousin qui a facilité notre entrée dans le site de relogement «Voltaire» (Saint- Denis) ainsi que Pierre Bergeron et Roxana Albu, responsables pour l'association Convivances, du site de l'avenue Pierre Sémard (Ivry-sur-Seine). Nous avons aussi mené des entretiens avec les Roms roumains relogés dans deux «Villages de l'espoir » où les familles n'ont plus le droit de faire la ferraille (Ivry-sur-Seine).

9. Nous avons préservé l'anonymat des personnes (et parfois les lieux), soit à leur demande expresse, soit parce que leur situation administrative difficile l'imposait. Les prises de vue ont toutes été faites avec l'accord des personnes photographiées.

10. Ceci confirme ce qu'écrit Martin Olivera, à savoir que ces familles roumaines « ne vivaient pas en bidonville au pays, pas plus qu'elles n'y subsistaient grâce au recyclage des ordures » (Olivera, 2015, p. 304). Par contre, dans les environs de Bucarest, certaines familles Rom fondaient l'aluminium de récupération pour le transformer en lingots (Hasdeu, 2009, p. 131).

11. Les Rudar sont souvent assimilés aux Roms roumains, or, d'après l'historien Petre Petcut, qui nous accompagnait lors de l'entretien, ils ne se reconnaissent ni comme Roms, ni comme Roumains.

12. Voir le site Urbarom, ainsi que les publications des membres de l'ANR Marg-In, celles de O. Legros, G. Cousin, M. Lièvre, M. Olivera et A. Clavé-Mercier.

13. Certains témoignages recueillis par les chercheurs de l'ANR Marg-In montrent cependant que l'activité de récupération n'est pas antinomique avec celle de la mendicité.

14. Un parallèle est intéressant à opérer avec les pratiques de cueillette décrites dans ce numéro dans les textes de S. Doyon et de M.A. Tareau et al.

15. https://www.senat.fr/questions/base/2012/qSEQ120700525.html

16. Au sens où l'emploie J.-C. Scott (2009, p. 199-218) qui analyse le braconnage dans les forêts par les paysans aux XVIII et $\mathrm{XIX}^{\mathrm{e}}$ siècles qui revendiquent le "droit » à utiliser les ressources offertes par la forêt, même lorsque cela leur est interdit.

17. Processus mis en évidence par les enquêtes des chercheurs de l'ANR Marg In.

18. O. Legros et M. Olivera analysent la relation entre les stratégies résidentielles des familles roms (contraintes par les politiques publiques, notamment les expulsions) et la constitution de réseaux sociaux essentiels sur le plan économique.

19. Ces représentations renvoient, de façon plus générale, aux stéréotypes et préjugés sur les Roms, minorité qui concentre le plus d'opinions négatives, très bien analysées dans l'article de $\mathrm{T}$. Vitale et al. (2018).

\section{RÉSUMÉS}

Les «ferraillous» sont des hommes, et parfois des femmes, qui collectent dans les rues les métaux abandonnés ou les « encombrants » qui, triés et bricolés, seront revendus aux grossistes. De la sorte, cette économie grise alimente les filières mondialisées et lucratives de la métallurgie. Boulot de la débrouille, glaner la ferraille est le fait de personnes en situation de marginalité 
sociale et économique qui réalisent leurs activités dans des marges urbaines, autant d'espaces ressources pour ces cueilleurs contemporains. Les entretiens réalisés dans les banlieues de Paris témoignent que, par leurs pratiques et savoir-faire, les ferraillous s'affranchissent des obstacles que leur situation personnelle et leur activité, plus ou moins tolérée, leur imposent. S’y révèle aussi une hiérarchie en leur sein ainsi que la place spécifique attribuée à la figure du Rom.

Scrap collectors are men or women who collect abandoned metals in the streets, which are sorted, tinkered, resold to wholesalers and, finaly, reintegrated into the metallurgy industry. Gleaning scrap is a do-it-yourself job, most often done by people in situations of economic and social precariousness. These contemporary gatherers realize their activities in urban margins, as many resource areas. The interviews conducted in the Paris suburbs show that, by their practices and know-how, they overcome the obstacles that their personal situation and their activity, more or less tolerated, impose. They also reveal a hierarchy within the scrap collectors and the specific place assigned to the Roma figure.

INDEX

Keywords : scrap dealer, wastepicker, Roma, suburb

Mots-clés : ferrailleur, récupérateur, Rom, marge, banlieue

Thèmes : Sur le Champ - Sur le Terrain

\section{AUTEURS}

\section{BÉNÉDICTE FLORIN}

Bénédicte Florin, benedicte.florin@univ-tours.fr, est maître de conférences en géographie à l'Université de Tours et chercheuse à l'UMR CITERES, équipe Monde Arabe et Musulman (EMAM). Avec Claudia Cirelli, elle est à l'origine de la création du réseau de recherche Sociétés Urbaines et déchets (https://sud.hypotheses.org/. Elle a récemment publié :

- Florin B., 2018. The Rag Pickers of Cairo: The Dregs of the City or "Garbage Businessmen"?. Ethnologie française, $\mathrm{n}^{\circ} 153$, p. 487-498.

- Florin B., 2016. De l'indignité à l'indignation : petites luttes, résistances quotidiennes et tentatives de mobilisation des récupérateurs de déchets à Istanbul. Cultures \& conflits, $\mathrm{n}^{\circ} 101$, p. 99-120. halshs-01333376v1

- Allix É., Florin B., 2016. Indésirables dans la ville, utiles dans l'ordure. Les récupérateurs de déchets au Maroc. Géographie et culture, n 96, p. 23-45 halshs-01718589v1

\section{PASCAL GARRET}

Pascal Garret, pascal.garret@bab-el-louk.org, est architecte, sociologue et photographe indépendant dans le cadre de recherches en sciences sociales. Ses travaux sont présentés sur le site http://www.bab-el-louk.org/ Il a récemment publié :

- Garret P., Florin B., 2018. Rien ne se perd, tout se récupère ! Pour une reconnaissance des récupérateurs informels de Casablanca. Urbanités [en ligne], octobre 2018. http://www.revueurbanites.fr/urbanites-africaines-garret-florin/

- Garret P., 2017. Une tournée de récupération à Istanbul. In Chevallier D., Tastevin Y.-P., (dir.), Vies d'Ordures. De l'économie des déchets. MuCEM et Artlys, p. 138-141. 